\title{
UMA ANÁLISE DOS DETERMINANTES DA TAXA DE CRIMES DE HOMICÍDIOS NOS ESTADOS DO BRASIL: UMA APLICAÇÃO EM PAINEL DINÂMICO
}

\section{RESUMO}

\author{
Antonio Marcos Pinto ${ }^{1}$ \\ Joedson Jales Farias ${ }^{2}$ \\ Rodolfo Ferreira Ribeiro da Costa ${ }^{3}$ \\ Francisco Soares de Lima $^{4}$
}

Estimar a taxa de crimes de homicídios para cada Estado brasileiro e verificar a relação do nível da criminalidade do período anterior e seus efeitos sobre o nível da criminalidade do período atual comporta o uso do modelo de dados em painel dinâmico. O aumento do número de crimes de homicídios dolosos está cada vez maior, de modo que, a taxa de crimes de homicídios defasada mostrou-se significativa e apresentou sinal esperado. O que significa dizer que a taxa de crimes de homicídios de um período passado influencia positivamente na taxa de crimes de homicídios do período atual. Analisando os sinais alcançados e comparando-os com os sinais esperados podemos citar as variáveis: taxa de crimes de homicídios defasada e escolaridade média: foram significativas e apresentaram sinal negativo, o que indica que quando esta variável aumenta a criminalidade diminui. Concluímos que os resultados alcançados se aproximam mais do esperado para o comportamento das variáveis utilizadas quando adotado o uso do MMG - Método dos Momentos Generalizados em Sistema, que se mostrou ser o melhor estimador para este estudo.

Palavras-chave: Painel dinâmico, Violência defasada, Taxa de homicídio doloso.

\begin{abstract}
To estimate the crime rate of homicides for each Brazilian state and to verify the relation of the level of crime of the previous period and its effects on the level of crime of the current period, involves the use of the data model in dynamic panel. The increase in the number of crimes of felonious homicides is increasing, so that the rate of homicide crimes lagged was significant and showed an expected signal. What it means to say that the crime rate of homicides of a past period positively influences the crime rate of homicides of the current period. Analyzing the signs obtained and comparing them with the expected signs, we can cite the variables: homicide crime rate lagged and average schooling: they were significant and showed a negative sign, which indicates that when this variable increases crime decreases. We conclude that the results obtained are closer than expected to the behavior of the variables used when adopting the MMG -

\footnotetext{
${ }^{1}$ Graduação em Ciências Econômicas (UERN). E-mail: antonio.marcospinto@hotmail.com

2 Doutorado em Rconomia (UFPE). Professor Adjunto (UERN). E-mail: joedsonfarias@uern.br

${ }^{3}$ Doutor em Economia pela Universidade Federal do Ceará (CAEN/UFC) e Pós-Doutor pela Universidade Federal do Rio Grande do Norte (UFRN), Professor da Universidade do Estado do Rio Grande do Norte. E-mail: rodolfocosta@uern.br

${ }^{4}$ Doutor em Economia pela Universidade Federal do Ceará (CAEN/UFC), Professor da Universidade do Estado do Rio Grande do Norte (UERN) e do Programa de Pós-Graduação em Economia (PPE/UERN). E-mail: fsoaresdelima@yahoo.com.br.
} 
Generalized Moments Method in the System, which proved to be the best estimator for this study.

Keywords: dynamic panel, outdated violence, intentional homicide rate.

\section{INTRODUÇÃO}

No Brasil o aumento da criminalidade tem chegado a um patamar nunca percebido antes, a quantidade de crimes tem causado prejuízos e desconfortos a toda a população, a atenção a este problema vem superando o foco até mesmo de outras problemáticas importantes como: inflação, fome, saúde e educação.

Esse trabalho tem por objetivo estudar o impacto dos gastos com segurança pública na taxa de homicídios, para isso avalia um Painel de Dados Dinâmicos de 2001 a 2014 relativos aos 26 Estados brasileiros e o Distrito Federal, na tentativa de relacionar as variáveis explicativas com a taxa de homicídios para explicar a existência da defasagem na violência.

Segundo Duenhas (2014, p. 181) "O conceito de violência, por sua vez, é mais amplo e envolve mais de uma dimensão, podendo assumir diferentes formas em épocas diferentes assim como diferentes entendimentos em sociedades distintas". Adotou-se o conceito de violência de Michaud (1989):

"[...] violência quando, numa situação de interação, um ou vários atores agem de maneira direta ou indireta, maciça ou esparsa, causando danos a uma ou a mais pessoas em graus variáveis, seja em sua integridade física, seja em sua integridade moral, em suas posses, ou em suas participações simbólicas e culturais (1989, p. 10-11)".

A preocupação com a criminalidade passa a ser um problema mais comum da sociedade brasileira que afeta todas as comunidades e interferem no seu modo de trabalhar e viver. Ao passo que a violência geralmente acompanha a criminalidade deixando-a ainda mais ofensiva e o seu dano acaba por gerar outros problemas ou atenuar algumas das preocupações dos Estados e cidades. O efeito negativo da criminalidade nas demais preocupações da sociedade a coloca em lugar de destaque e no centro das atenções de um modo geral. No entanto, nem todo crime é acompanhado pela violência, por exemplo: o crime de falsificação de documentos para fazer a 
declaração do imposto de renda ainda que seja ilegal não é acompanhado por violência.

Esse aumento desenfreado da incidência de crimes tem causado importantes perdas no setor econômico de um país e é capaz de afetar indicadores sociais, prejudicando a atividade econômica como um todo. O aumento da mortalidade causado pela violência na forma de crime reduz a expectativa de vida dos brasileiros, gera gastos excessivos na saúde e recruta os jovens da escola proporcionando um salto na evasão escolar.

Não podemos tratar a criminalidade como somente um caso de polícia. Muitas vezes a origem do ato delituoso é a própria situação que o infrator decide ou não cometer o crime, que ao mesmo tempo, reflete diretamente na economia de um país. Nesta perspectiva a presença da criminalidade no cotidiano da população brasileira se torna um problema social de grande importância a ser estudado.

A evolução das sociedades de hoje é acompanhada pelo desenvolvimento econômico, garantia de direitos da população, e também pelo aumento da criminalidade e violência. É um desafio enorme entender a relação e a contribuição de cada fator para este fato está acontecendo cada vez mais frequente, é preciso identificar os fatores que contribuem para o aumento da criminalidade e também a participação da violência cada vez mais comum no momento do cometimento de crimes.

Para prevenir o crime é muito importante conhecer e entende-lo. A crescente sensação de insegurança chama a atenção de instituições e órgãos responsáveis pela segurança da população. O Estado tenta coibir e prevenir essa conduta que atinge diretamente a qualidade de vida das pessoas. Na maioria das vezes a violência está presente de forma decisiva na ação criminosa fazendo com que a população mude de hábitos do dia-a-dia tentando reduzir a incidência de crimes a que estão expostos.

O aumento do investimento em Segurança Pública tem sido a política mais utilizada nos dias de hoje para coibir essa crescente criminalidade, no entanto, não há ainda um entendimento entre os formadores de Políticas Públicas referentes à melhor maneira de resolver este problema. 
Por tanto, este trabalho busca identificar se a taxa de crimes de homicídios por (100.000) habitantes é influenciada pelos Gastos Públicos com Segurança Pública por pessoa e a relação entre essas variáveis com a atividade econômica. Busca-se avaliar a existência de elos dinâmicos na taxa de crimes por meio de um painel dinâmico.

O crime pode ser considerado um problema econômico porque para acontecer depende um lado da condição econômica do agente ou da vítima e, do outro, seu efeito reduz a possibilidade de desenvolvimento da economia. $\mathrm{O}$ crime também assume um aspecto político, devido demandar recursos públicos e decisões de agentes formadores de mecanismos em detrimento de outras ações alheias à própria criminalidade.

Entender o crime passa a ser o pontapé inicial na tentativa de coibi-lo. Por isso, a criação de mecanismos novos e o desenvolvimento das formas existentes de prevenção do crime não deixando para agir apenas como repressor da atividade criminosa passa a ser a metodologia usada em quase todas as sociedades.

A produção de conhecimento fornece insights para as ações das polícias, buscando reduzir os entraves que nublam as ações de planejamento e diagnósticos que possibilitam a avaliação do problema como um todo. As informações que chegam aos órgãos de Segurança Pública são ferramentas que necessitam serem incentivadas e compreendidas. É preciso investir em inteligência dos órgãos responsáveis e na organização e coleta de estatística das polícias para melhor combater o crime. Números da incidência por local de ocorrência de crimes pode ser usado para designar e direcionar recursos ideais para cada situação e local.

\section{MODELO ECONÔMICO DO CRIME}

Para o desenvolvimento deste trabalho será abordado duas correntes de pensamentos: a primeira é que os fatores determinantes no combate a violência são a intervenção policial e repressão judicial; a segunda é a que tem o papel do ambiente econômico e social do indivíduo como principal fator explicativo do comportamento criminoso, com mais atenção ao grau de informalidade da população; mostrando atenção especial a violência defasada (áreas atingidas pela violência no passado tendem a continuarem sendo atingidas pela violência nos dias atuais, mesmo com os 
esforços das autoridades em evitar a violência mas esta não desaparece de imediato por completo).

Com o crescente aumento da criminalidade cresce também a preocupação com os seus efeitos, por isso, tentamos construir hipóteses baseadas em teorias e modelos que buscam direcionar esforços aplicados à realidade feita na forma de testes de informações disponíveis.

O emprego de medidas para combater a criminalidade não apresenta um resultado imediato, uma área atingida pela violência não deixa de ser violenta de uma hora para outra. Dessa forma é possível citar a presença de uma defasagem da violência à medida que ela é combatida.

É mais comum encontrar trabalhos deste tipo proveniente de autores e estudiosos sociais, em uma breve revisão das teorias que relaciona as variáveis socioeconômicas: grau de informalidade e a violência defasada, em uma dada região, quando somada a dados elaborado pelo meio social é possível ir muito além do discurso da pobreza e do discurso da impunidade comumente usados para justificar 0 ato criminoso das pessoas.

No Brasil a incidência de crimes vem preocupando a população de forma a mudar sua forma de viver. Comerciantes e trabalhadores são vítimas diárias da crescente criminalidade que na maioria das vezes é acompanhada de imensa violência por parte dos criminosos. Cada vez mais nos deparamos com relatos de vítimas de diversos tipos de crimes. Dessa forma, a atuação das autoridades repressoras do crime da Secretaria Estadual de Segurança Pública e Defesa Social como às Polícias Militar e Civil, são forçadas a evoluírem suas formas de lidar com esta realidade ao ponto de procurarem modernizar as suas ações na luta contra o crime. A abordagem nos dias atuais se dá de maneira a prevenir a ação criminosa intensificando operações e com estas buscando resultados mais eficazes de combate à atividade delituosa. De modo que, o que se procura é evitar o acontecimento do fato já que após ele ocorrido se torna difícil reverter os resultados da ação do criminoso.

A dificuldade a ser enfrentada é conhecer os incentivos e os pontos negativos que os infratores usam para a tomada de decisões em cometer ou não o crime. Outro 
ponto, a condição do meio social do indivíduo, as questões socioeconômicas das pessoas influenciam no modo como elas vivem e pode também determinar se vão ou não cometer algum crime.

Essa grande gama de inter-relacionamento de fatores que influenciam e são influenciados pela atitude delituosa constituem a rede de fatores socioeconômicos na qual o crime se apresenta, de forma geral, uma externalidade negativa do desenvolvimento socioeconômico. (Leonardo, 2007, p.25).

Para estimar os determinantes do nível de crimes de homicídios nos Estados brasileiros no período de 2001 a 2014 é necessário mensurar os efeitos dinâmicos do nível de crimes de homicídios; mostrar os efeitos de políticas públicas de segurança nos indicadores de criminalidade; verificar os efeitos de indicadores sociais sobre a ocorrência de crimes; observar o efeito das variáveis do mercado de trabalho na ocorrência de crimes de homicídios. O Estudo propõe trabalhar com um painel de dados dinâmicos. A hipótese é de que existe um componente inercial na ocorrência de crimes, em particular os crimes de homicídios.

$\mathrm{Na}$ mesma lei que rege o Código Penal Brasileiro a definição de crime literalmente é: fato típico, antijurídico e culpável Decreto Lei 2.848 (1940).

A literatura econômica do crime foi desenvolvida por Gary Becker. Em seu trabalho Becker impôs um marco à abordagem sobre os determinantes da criminalidade ao desenvolver um modelo formal em que o ato criminoso decorreria de uma avaliação racional em torno dos benefícios e custos esperados aí envolvidos, comparados aos resultados da alocação de seu tempo no mercado de trabalho legal.

Segundo Becker (1968) o criminoso é um agente racional e o crime ocorre se o benefício recebido por cometer o crime for superior ao custo de se cometer o crime.

Ainda de acordo com Becker (1968), citado por César e Mariana (2013, p. 6384), "o prejuízo social do crime é nada menos que a simples subtração da lesão social do crime pelo benefício auferido pelo indivíduo que cometeu o crime. O benefício marginal conseguido pelo criminoso é uma função decrescente, acompanhado pelo fato de que, a lesão social do crime cresça com o aumento do nível de crime. Então o prejuízo social do crime é crescente". 
Então a função fornecida por Becker fica assim:

$$
H_{i}=H_{i}\left(O_{i}\right)
$$

onde

$$
H_{i}=\frac{d H i}{d O i}>0
$$

$\mathrm{Na}$ equação acima, $H_{i}$ significa a lesão ocasionada por determinada atividade ilícita, e $O_{i}$ mostra o nível/quantidade dessa atividade.

Dessa forma, observando o prejuízo marginal da sociedade causado pelo crime, podemos notar que o benefício alcançado pelo criminoso tende a aumentar com a quantidade de delitos.

$$
G=G(O)
$$

onde

$$
G^{\prime}=\frac{d G}{d O}>0
$$

Ao explicar o seu Modelo Econômico do Crime, Becker Afirma que: para determinar como combater o crime de uma forma ideal é muito importante desenvolver um modelo para incorporar as relações comportamentais por trás dos custos econômicos do crime. Estas relações podem ser divididas em cinco categorias: (1) as relações entre o número de crimes, chamados "delitos" e o custo de cometer o crime, (2) o número de crimes e o castigo imposto por ter cometido crime, (3) o número de crimes, detenções e condenações e as despesas públicas sobre a polícia e os tribunais para combater o crime, (4) o número de condenações e os custos de prisão ou outros tipos de punições; (5) o número de crimes e as despesas privadas em matéria de proteção e apreensão". (Becker apud César e Mariana, 2013 p. 172).

Em conjunto com o prejuízo social é preciso analisar os custos do Estado, quanto mais crimes acontecerem maiores serão os custos para combatê-los. O custo marginal nada mais é que a derivada dos custos em função do número de crimes e este será maior que zero.

$$
\begin{aligned}
& C=C(A) \\
& C^{\prime}=\frac{d C}{d A}>0
\end{aligned}
$$

O aparato usado pelo Estado para combater o crime pode ser determinado com " $A$ ", que depende de materiais, recursos humanos e capital. 
Então:

$$
A=f(m, r, c)
$$

A taxa de condenação em relação ao número de crimes serve para determinar os custos alcançados pelo Estado, de modo, o aumento no custo da execução da pena proveniente da condenação explica o custo total do crime.

O nível de " $A$ " depende do sucesso da condenação do criminoso. $A \cong p O$, sendo " $p$ " o percentual de condenação; desse modo o aumento de " $p$ " ou de " $O$ " provocará um aumento nos custos da prisão e condenação dos delinquentes.

\section{METODOLOGIA}

O trabalho utiliza dados oficiais ao nível das unidades federativas, coletados em diversas fontes. A estrutura dos dados compõe um painel com 27 unidades da federação para o período de 2001 a 2014.

Especificamente, as informações foram retiradas junto ao IBGE, a Pesquisa Nacional por Amostras de Domicílios (PNAD); do Instituto de Pesquisa Econômica Aplicada (IPEA), ao Mapa da Violência, DATASUS do Ministério da Saúde, Secretaria Nacional de Segurança Pública (SENASP), Ministério da Justiça (MJ) e Ministério da Fazenda (MF).

A taxa de crimes de homicídios: foi retirada do Sistema de informação sobre Mortalidade do Ministério da Saúde. Essa taxa é específica para o tipo de óbito por 100.000 habitantes. Essa variável apresenta o número de óbitos por homicídios para cada unidade da federação relativa ao período de 2001 a 2014.

Renda per capita: Essa variável representa o rendimento médio por pessoa que a população recebe como recompensa pelo seu trabalho em forma de salário, é importante utilizar essa variável no trabalho por que na literatura econômica se espera que quanto maior a renda recebida por um indivíduo, menos será as chances de ele ingressar no mundo do crime. Os dados relativos ao rendimento médio por pessoa foram retirados do banco de dados do IPEADATA com base na Pesquisa Nacional por Amostra de Domicílios (Pnad) do IBGE referente ao período de 2001 a 2014. 
Taxa de Escolaridade: essa variável representa a parte da população que tem mais de 15 anos de escolaridade. Estes dados foram retirados do banco de dados do IPEADATA para o período de 2001 a 2012.

Coeficiente de Gini: os dados desta variável foram retirados do banco de dados do IPEADATA com base na Pesquisa Nacional por Amostra de Domicílios (Pnad) do IBGE para o período de 2001 a 2012.

Gastos Públicos com Segurança Pública per capita: os dados desta variável foram retirados do banco de dados do IPEADATA com base na Pesquisa Nacional por Amostra de Domicílios (Pnad) do IBGE para o período de 2001 a 2012, e representa o Gasto Público com segurança por pessoa para cada Estado brasileiro.

Gasto Público com Assistência Social: os dados desta variável foram retirados do banco de dados do IPEADATA com base na Pesquisa Nacional por Amostra de Domicílios (Pnad) do IBGE para o período de 2004 a 2012, e representa o Gasto Público com Assistência Social por pessoa para cada Estado brasileiro.

Taxa da pobreza: os dados desta variável foram retirados do banco de dados do IPEADATA com base na Pesquisa Nacional por Amostra de Domicílios (Pnad) do IBGE para o período de 2001 a 2014, e representa o percentual de pessoas em domicílios com renda domiciliar por pessoa inferior a linha da pobreza. A linha da pobreza no Brasil é considerada o dobro da linha da extrema pobreza, já que a extrema pobreza é de $R \$ 70,00$ por pessoa, a linha da pobreza é de $R \$ 140,00$ por pessoa.

O Grau de informalidade: foi obtido do banco de dados do IPEADATA com base na Pesquisa Nacional por Amostra de Domicílios (Pnad) do IBGE, corresponde ao resultado da seguinte divisão: empregados sem carteira mais trabalhadores por conta própria dividido pelos trabalhadores protegidos mais empregados sem carteira mais trabalhadores por conta própria; para o período de 2001 a 2014. Importante citar que o IPEA efetua este cálculo de três maneiras distintas, para esse estudo utilizamos o grau de informalidade tipo 1, diferenciado dos demais maneiras por usar as informações citadas anteriormente na execução do cálculo.

Densidade demográfica: os dados desta variável foram retirados do banco de dados do IPEADATA com base na Pesquisa Nacional por Amostra de Domicílios (Pnad) 
do IBGE para o período de 2004 a 2012, e representa a quantidade de habitantes por quilômetros quadrados para cada uma das 27 unidades federativas do Brasil.

Tabela 1 - Variáveis e fontes.

\begin{tabular}{l|l}
\multicolumn{1}{c|}{ DESCRIÇÃO } & FONTE \\
\hline Taxa dee crimes de homicídios dolosos por 100.000 habitantes & SENASP-MJ \\
Coeficiente de Gini & IPEA/PNAD \\
Renda domiciliar per capita & IPEA/PNAD \\
Taxa da pobreza & IPEA/PNAD \\
Taxa de escolaridade média & IPEA/PNAD \\
Gastos públicos com segurança per capita & IPEA/PNAD \\
Gastos públicos com assistência social per capita & IPEA/PNAD \\
Densidade demográfica & IBGE \\
Grau de informalidade & IPEA/PNAD \\
\hline
\end{tabular}

Fonte: Elaborado pelo autor.

\subsection{MODELO DE DADOS EM PAINEL}

A abordagem para lidar com dados disponíveis em unidades cross-section ao longo do tempo, também chamados de dados longitudinais, é a de dados em painel. Os modelos de dados em painel se dividem em efeitos fixos e efeitos aleatórios. Formalmente, temos o seguinte:

$$
\begin{gathered}
y_{i}=\alpha_{i}+\beta^{\prime} x_{i t}+u_{i t} \\
i=1,2, \ldots, N \\
t=1,2, \ldots, T
\end{gathered}
$$

Onde $y_{i t}$ é a variável dependente, $x_{i t}$ é o vetor de variáveis explicativas da iésima unidade cross-section, no tempo $t$. $\alpha_{i t}$ captura elementos específicos à i-ésima unidade cross-section, que supõe-se invariável ao longo do tempo.

\subsection{MODELO DE DADOS EM PAINEL DINÂMICO}

Um dos métodos bastante utilizados para a estimação é o Modelo de Dados em Painel Dinâmico. Quando mencionamos esse tipo de modelagem temos que distinguir entre dois tipos: 


\section{O Modelo de Correlação Serial}

$$
\begin{aligned}
& y_{i t}=\rho x_{i t}+\alpha_{i}+w_{i t} \\
& w_{i t}=\rho w_{i, t-1}+\mu_{i t}|\rho|<1
\end{aligned}
$$

\section{O Modelo de Estado Dependente}

$$
y_{i t}=\beta x_{i, t-1}+\gamma y_{i, t-1}+\alpha_{i}+\mu_{i t}
$$

Em ambos os casos temos que $\alpha_{i} \sim \boldsymbol{I N}\left(\mathbf{0}, \sigma_{\alpha}^{2}\right)$ e $\boldsymbol{\mu}_{\mathrm{i}} \sim \boldsymbol{I N}\left(\mathbf{0}, \boldsymbol{\sigma}_{\mu}^{2}\right)$ e $\alpha_{i}$ e $\boldsymbol{\mu}_{i}$ são dependentes.

Além disso, quando consideramos o método de estimação máxima verossimilhança, temos que considerar a função de verossimilhança condicional (condicional ao valor inicial de $y_{i 0}$ ) e a função de verossimilhança não condicional. No segundo caso assumimos que o processo tem sentido por um longo tempo e que $y_{i 0}$ é uma observação da distribuição de equilíbrio de $y_{i 0}$.

Em todos os modelos de componentes de variantes há o problema de obter estimativas de $\sigma_{\alpha}^{2}$ e $\sigma_{\mu}^{2}$. Se ai depende dos valores médios de $x_{i t}$. Nerlove (1998) argumenta contra o uso de um método sugerido por Greene e Judge et al. em seus textos de econometria e afirma que ele é utilizado em vários programas de computador porque produz estimativas negativas dos componentes variantes e induz as rejeições desnecessárias do modelo.

Uma vez que as estimativas dos componentes variantes tenham sido obtidas, trata-se do método de estimação do modelo da equação com relação ao modelo podemos estimar primeiro o modelo pelo método MQVD:

$$
\begin{gathered}
\alpha_{i}=\pi^{\prime} \bar{x}_{i}+w_{i} \\
y_{i t}=\pi^{\prime} \bar{x}_{l}+\beta^{\prime} x_{i t}+w_{i}+\mu_{i t}
\end{gathered}
$$

\subsection{MÉTODO DOS MOMENTOS GENERALIZADOS}

Em econometria, o método dos momentos generalizado (MMG) é um método genérico para estimar parâmetros em modelos estatísticos. Normalmente ela é aplicada no âmbito de modelos semi-paramétricos, em que o parâmetro de interesse é 
de dimensão finita, ao passo que a forma completa da função de distribuição dos dados pode não ser conhecida e por conseguinte, a estimativa de probabilidade máxima não é aplicável .

O método requer que um certo número de condições de momentos que foram especificados para o modelo. Estas condições de momento são funções dos parâmetros do modelo e os dados, de modo que sua expectativa é de zero nos verdadeiros valores dos parâmetros. O método MMG, em seguida, minimiza uma determinada norma das médias amostrais das condições de momento.

Os estimadores MMG são conhecidos por ser consistente, assintoticamente normal, e eficiente na classe de todos os avaliadores que não utilizam de alguma informação extra além do que consta nas condições de momento.

O método dos momentos generalizado foi introduzido por (Lars Hansen 1982). A exposição aqui baseia-se em Hayashi (2000). A equação a ser estimada é, em notação matricial, $y=X \beta+\mu$ (1) com a linha típica $y_{i}=X_{i} \beta+\mu$, a matriz de regressores $X$ é $N x K$, onde $n$ é o número de observações. Alguns dos regressores são endógenos. $\mathrm{O}$ conjunto de variáveis instrumentais é $Z$ e $n x L$. Este é o conjunto completo de variáveis que estão a ser assumida exógeno, isto é, $E($ Ziui $)=0$. Nos proporciona os instrumentos em [ $Z 1 Z 2]$, em que os instrumentos $L 1 Z 1$ são excluídos os instrumentos e os restantes $\equiv(L-L 1)$ instrumentos $L 2 Z 2 \equiv X 2$ são os instrumentos incluídos / regressores exógenos:

Regressores $X=\left[\begin{array}{ll}X 1 & X 2\end{array}\right]=[X 1 Z 2]=[$ endógena exógena $]$

Instrumentos $Z=[Z 1 Z 2]=[$ Excluído incluído $]$

A condição para que a identificação da equação é $L \geq K$ implicando que deve haver pelo menos instrumentos excluídos ( $L 1)$ como existem regressores endógenos (K1) como $Z 2$ é comum a ambas as listas. Se $L=K$, a equação é dita para ser exatamente identificada pela condição de ordem; Se $G>K$, a equação é "Overidentified". A condição de ordem é necessária mas não suficiente para a identificação.

A suposição de que os instrumentos $Z$ são exógeno pode ser considerada MMG linear apenas, e neste caso os instrumentos $L$ dar-nos um conjunto de $L$ momentos. A 
exogeneidade dos instrumentos significa que existem $G$ momentos ou condições de ortogonalidade, que será satisfeito no verdadeiro valor de $\beta$. Cada uma das equações em um momento $L$ corresponde a um momento de amostra. Para algum dado estimador de $\beta$, podemos escrever esses momentos $L$ amostra como:

$$
\begin{aligned}
& g(\beta)=1 \\
& y_{i}=x_{i} \beta+\mu_{i} \\
& y=[X 1 X 2][\beta 01 \beta 02] 0+\mu
\end{aligned}
$$

A intuição por trás MMG é escolher um estimador de $\beta$ que traz $g(\beta) \circ$ mais próximo possível de zero. Se a equação a ser estimada é exatamente identificada, de modo que $L=K$, então temos tantas condições, como momento equações de $L$ fazemos incógnitas: o coeficientes $K$ em $\beta$. Neste caso, é possível encontrar um $\beta$ que resolve $g(\beta)=0$.

\subsection{MODELO ESTIMADO}

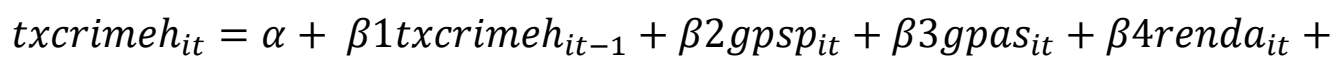

$\beta 5$ esc $_{i t} \beta 6$ txpb $_{i t}+\beta 7$ cgini $_{i t}+\beta 8$ ginf $_{i t}+\beta 9$ densi $_{i t}+\mu_{i t} v_{i t}$

Onde:

txcrimeh = taxa de crimes de homicídios

gpsp = gastos públicos com segurança pública

gpas = gastos públicos com assistência social

renda $=$ renda per-capita

esc $=$ taxa de escolaridade

txpb = taxa da pobreza

cgini $=$ coeficiente de gini

ginf = grau de informalidade da força de trabalho 
densi $=$ densidade demográfica

$\mu=$ representa os efeitos que refletem características não observadas de cada estado

$v$ = termo de distúrbio aleatório típico

A variável escolhida para explicar a criminalidade é a taxa de homicídios dolosos por cem mil habitantes. Já as variáveis explicativas consideradas no estudo em questão procuram refletir os fatores condicionantes da violência gerada pelos crimes, que detém a atenção do Estado na tentativa de combater e reduzir esses índices.

Assim foram incluídas variáveis que representam as condições socioeconômicas, demográficas e do sistema de segurança pública e defesa social e assistência social.

\section{RESULTADOS}

Implementamos o modelo de dados em painel dinâmico. Segue os resultados e análise do modelo estimado. A seguir, vamos mostrar como ficam os resultados das estimações dessas variáveis, aplicando o comando painel de dados dinâmicos.

Tabela 2 - Modelo estimado MMG sistemas.

\begin{tabular}{l|rrrr}
\hline Taxa de crimes de homicídios & \multicolumn{1}{l}{ Coeficiente } & Desvio padrão & \multicolumn{1}{c}{$\mathrm{t}$} & $\mathrm{P}>\mathrm{t}$ \\
\hline taxa de crimes de homicídios em t-1 & 0.6883839 & 0.0674824 & 10.2 & 0.000 \\
& & & & \\
gastos com segurança pública & -0.0069948 & 0.0085676 & -0.82 & 0.416 \\
gastos com assistência social & 0.0041537 & 0.0057682 & 0.72 & 0.473 \\
renda média & 0.0179454 & 0.008518 & 2.11 & 0.038 \\
escolaridade média & -5.232 .265 & 2.315 .043 & -2.26 & 0.026 \\
taxa da pobreza & -0.1738402 & 0.1491077 & -1.17 & 0.246 \\
coeficiente de gini & 0.5150301 & 1.883 .718 & 0.03 & 0.978 \\
grau de informalidade & -0.2600457 & 0.1591418 & -1.63 & 0.105 \\
densidade demográfica & -0.0532691 & 0.0727652 & -0.73 & 0.466 \\
\hline Fonte: & & & &
\end{tabular}

Fonte: elaborado pelo autor.

A tabela 2 acima representa as estimativas do modelo dados em painel dinâmico para as variáveis selecionadas correspondentes ao período observado pelo trabalho. $\mathrm{Na}$ coluna 1 descreve-se as variáveis; na coluna 2 temos os coeficientes estimados, na coluna 3 vem o desvio padrão, na coluna 4 os valores de "t" e na coluna 5 temos os 
valores de $P>t$, esses valores de $P>t$ são usados para determinar se a variável explicativa tem ou não significância.

Para justificar se a variável é explicativa para a taxa de crimes de homicídios devemos observar se ela é significativa ao nível de $5 \%$, o sinal do coeficiente informa em qual direção a taxa de crimes de homicídios se move; se positivo a relação é direta, se negativo a relação é inversa.

A variável taxa de crimes de homicídios defasada mostrou-se significativa e apresentou sinal esperado. O que significa dizer que a taxa de crimes de homicídios de um período passado influencia positivamente na taxa de crimes de homicídios do período atual.

A variável escolaridade mostrou-se significativa e apresentou sinal esperado. $\mathrm{O}$ que sugere uma relação inversa do nível de escolaridade da população com a criminalidade. Isso significa que a taxa de crimes de homicídios sofre um efeito redutor quando aumentamos a escolaridade das pessoas.

A variável gastos com segurança pública mostrou-se não significativa apesar de apresentar sinal esperado.

A variável taxa da pobreza mostrou-se não significativa e apresentou sinal esperado.

O grau de informalidade mostrou-se significativo apenas ao nível de $10 \%$ e apresentou sinal esperado. $O$ que indica que o aumento no grau de informalidade não explica a criminalidade.

A variável densidade demográfica apresentou-se não significativa apesar de sinal esperado.

A variável gastos com assistência social mostrou-se não significativa e apresentou sinal positivo, diferente do esperado.

A variável renda per capita mostrou ser significativa e apresentou sinal positivo, diferente do esperado, o que indica que o nível de renda das pessoas pode ser fator influenciador do aumento da criminalidade. 
O coeficiente de gini apresentou-se não significativo e com sinal positivo. O que indica que o coeficiente de gini não explica a taxa de crimes de homicídios neste trabalho.

\section{CONCLUSÃO}

Os dados levantados de cada Estado e arrumados em painel dinâmico referentes ao período de 2001 a 2014 estimou a taxa de crimes de homicídios para cada Estado brasileiro avaliando especificamente os efeitos da inércia da taxa de crimes de homicídios do período t-1 no período atual.

Os resultados sugerem um significativo componente inercial na taxa de crimes de homicídios. Isso significa que a taxa de crimes de homicídios tem como determinante a taxa de homicídios no período anterior, conforme foi possível observar após estimar as variáveis usadas nesse estudo.

Com base na literatura econômica aplicada em trabalhos anteriores e replicada neste estudo nos possibilitou entender a relação de cada uma dessas variáveis com a taxa de crime de homicídios.

O modelo econômico do crime utilizado sugere que os resultados obtidos tornam possível o entendimento dos determinantes do nível de crimes de homicídios no Brasil.

As variáveis renda média e escolaridade média revelaram-se significativas, no entanto, apenas a escolaridade média apresentou sinal negativo. Esse fato sugere que estas duas variáveis explicam a criminalidade, mas, somente a escolaridade influência de forma a reduzir a criminalidade.

O coeficiente positivo das variáveis renda média indica que quando ocorrer um aumento desta variável esse aumento será acompanhado pelo aumento da criminalidade, fato que sugere a necessidade de uma análise mais profunda dos resultados alcançados neste trabalho.

Além disso, o uso do MMG - método dos momentos generalizados em primeira diferença mostrou-se ser apropriado. Apresentando um estimador para a defasagem da 
violência robusto. No entanto, fez-se necessário usar o método dos momentos generalizados em sistema. (Arellano e Bond, 1991) citado por Kume (2013).

Então, concluímos que a respeitos dos dados disponíveis vê-se que as instituições públicas podem melhorar os padrões estabelecidos, revelando uma integração entre os órgãos insuficiente. A importância dessa integração entre os órgãos não seria benefício apenas para as instituições de segurança pública mas para todas as demais instituições públicas que tem por fim melhorar a sua eficiência e o bem estar da sociedade.

\section{REFERÊNCIAS}

CARLOS, Jorge Adriano. 0 crime segundo a perspectiva de Durkheim. Disponível em: <http://www.karlmarx.pro.br/apostilas/sociologia/emille_durkhein.pdf> Acesso em: 06 Fev 2016

CÓDIGO PENAL BRASILEIRO. Disponível em:<<http://www.planalto.gov.br/ccivil_03/decreto-lei/del2848.htm\#art361 >> Acesso em: 06 Fev 2016

INSTITUTO BRASILEIRO DE GEOGRAFIA E ESTATÍSTICA. Disponível em: $<<h t t p: / / w w w . c i d a d e s . i b g e . g o v . b r / x t r a s / p e r f i l . p h p ? l a n g=\&$ codmun=240800\&search=riogrande-do-norte|mossoro|infograficos:-informacoes-completas >> Acesso em: $02 \mathrm{Abr}$ 2016

JÚNIOR, Ledimar Fernandes. A Criminalidade no Brasil: avaliação do impacto dos Investimentos públicos e dos fatores socioeconômicos. Universidade do Estado do Estado do Rio Grande do Norte. Monografia do Curso de Graduação em Ciências Econômicas, Mossoró, 2014.

ARAÚJO, Jr., A. F. de. Raízes econômicas da criminalidade violenta no Brasil: um estudo usando micro dados e pseudopainel - 1981/1996. Revista de Economia e Administração. V. 1, n 3. Jul/set. p. 1-34, 2002.

ARAÚJO, Jr., A. F. e FAJNYLBER, P.. O Que Causa a Criminalidade Violenta no Brasil?: Uma Análise a partir do Modelo Econômico do Crime: 1981 a 1996. CEDEPLAR/UFMG, 2001.

SOUZA, Leonardo da Silva. Criminalidade, inteligência policial e economia: um estudo de relação de variáveis econômicas e a incidência de crimes na cidade de 
Florianópolis. Universidade Federal de Santa Catarina. Monografia do Curso de Graduação em Ciências Econômicas, Florianópolis, 2007.

SECRETARIA DE SEGURANÇA PÚBLICA E DA DEFESA SOCIAL. Centro Integrado de Operações de Segurança Pública. Subcoordenadoria de estatística e análise criminal. Disponível em:

<<http://adcon.rn.gov.br/ACERVO/SESED_RN/DOC/DOC000000000032335.PDF>> Acesso em: 02 Abr 2016.

SCHAEFER, Gilberto José; SHIKIDA, Pery Francisco Assis. Economia do Crime: elementos teóricos e evidências empíricas. Revista Analise Econômica, Ed. n³6, Ano 19, Setembro de 2001.

SOARES, Luiz Eduardo. Meu Casaco de general: $\mathbf{5 0 0}$ dias no front da Segurança Pública do Rio de Janeiro. Companhia das Letras. São Paulo, 2000.

IPEADATA. Disponível em: <http://www.ipeadata.gov.br> Acesso em: 15 Abr 2016.

BAUM, Christopher F. Enhanced routines for instrumental variables/MMG estimation and testing.

ESTANISLAU, César Vale; MORAIS, Mariana Teodoro. CONTRIBUIÇÕES DA ANÁLISE ECONÔMICA DO DIREITO PARA A POLÍTICA CRIMINAL. Revista do CAAP- Centro Acadêmico Afonso Pena/ Belo Horizonte n. 2/ v. XIX/ p. 63 - 84. 2013.

WAISELFISZ, Julio Jacobo. Mapa da Violência dos Municípios Brasileiros.

Organização dos Estados Ibero-Americanos para Educação, Ciência e Cultura. 1ạ Edição, 2007.

BECKER, Gary S. Crime and Punishment: An Economic Approach. Journal of Political Economics, v. 76, n. 2, mar - abril, pp. 169 - 217, 1968.

MADDALA, G. S. Introdução a econometria. $3^{\underline{a}}$ ed. LTC. Rio de Janeiro, 2003. cap. 15/ p.308 a 313.

KUME, Leandro. Uma estimativa dos determinantes da taxa de criminalidade brasileira: uma aplicação em painel dinâmico. Disponível em:

<<www.observatorioseguranca.org/pdf/01\%20(31).pdf>> Acesso em: 09 mai 2016.

Recebido em: junho de 2018

Aceito em: dezembro de 2018 